\title{
$X$. On the law of distribution of energy
}

\section{S. H. Burbury F.R.S.}

To cite this article: S. H. Burbury F.R.S. (1894) X. On the law of distribution of energy, Philosophical Magazine Series 5, 37:224, 143-158, DOI: 10.1080/14786449408620528

To link to this article: http://dx.doi.org/10.1080/14786449408620528

$$
\text { 曲 Published online: } 08 \text { May } 2009 .
$$

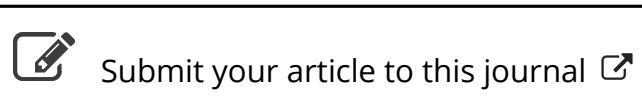

\footnotetext{
Llll Article views: 3
}

Q View related articles $\square$

Citing articles: 1 View citing articles $\sqsubset$ 
of field, the magnetic-twist curve for nickel is exactly similar to the same for iron. The reason is simply that with nickel there is a field of maximum twist for each value of current passing along the wire, although there is no field of maximum elongation. The explanation of this is given in my paper of 1888 (Trans. R. S. E. vol. xxxv.). It is sufficient to note that the twist, under a given combination of circular and longitudinal magnetizing forces, depends not only upon the elongations but also upon some function of these forces which changes sign with each, and to which the existence of the maximum twist is largely or altogether due. For even in the case of iron, which has a maximum elongation, the maximum twist occurs in quite a different field. Indeed the field of maximum twist varies with the value of the current along the wire.

Meanwhile the broad character of the hysteresis in the magnetic-elongation cycle, as established by Mr. Nagaoka's delicate experiments, agrees perfectly with what might be inferred from the character of the hysteresis in the magnetictwist cycle-a phenomenon whose experimental study is one of the simplest in the whole subject of magnetic strains.

Edinburgh University,

October 28, 1893.

X. On the Law of Distribution of Energy. By S. H. Burbury, F.R.S.**

$I^{\mathrm{F}}$ there be in any space a great number of mutually acting molecules, Boltzmann's law of distribution of energy requires that the number per unit of volume of molecules whose coordinates and momenta lie between assigned limits varies as $e^{-h(x+T)}$ in the known notation. The proofs of that law given by Boltzmann and Watson are based on the hypothesis that, from the instant when mutual action commences between two molecules to the instant when it ceases, no action takes place between either of them and any third molecule, or, as it is called, the encounters are binary. I propose to consider the more general case when, for instance, no group of molecules is ever free from the action of other parts of the system.

\section{PART I.}

(1) The following is a known proposition in the theory of Least Squares.

Let the chance of a certain magnitude lying between $x$ and $x+d x$ be $f^{\prime}(x) d x, f(x)$ being a function which vanishes

* Communicated by the Author. 
for infinite values of $x$, and is not altered by changing the sign of $x$, so that

$$
\begin{aligned}
& \int_{-\infty}^{\infty} f(x) d x=1 . \\
& \int_{-\infty}^{\infty} f(x) x d x=0 .
\end{aligned}
$$

Let

$$
\int_{-\infty}^{\infty} f(x) x^{2} d x=\overline{x^{2}}
$$

Let there be $\mathrm{N}$, a very great number, of such magnitudes, each independent of all the others, denoted by $x_{1}, x_{2} \ldots x_{\mathrm{N}}$.

Let it be required to find the chance that

$$
\frac{1}{\sqrt{\mathrm{N}}}\left(x_{1}+x_{2}+\ldots+x_{\mathrm{N}}\right)
$$

shall lie between $c$ and $c+d c$, and let this chance be $F(c) d c$. Then by the known method we find

$$
\mathrm{F}(c)=\mathrm{C} \int_{-\infty}^{\infty} d \theta \mathrm{X}^{\mathrm{N}} \epsilon^{-c \theta \mathrm{N}=1}
$$

in which $\mathrm{C}$ is constant and

$$
\begin{aligned}
\mathrm{X}= & \int_{-\infty}^{\infty} d x f(x) \epsilon^{\frac{x \theta \sqrt{-1}}{\sqrt{\bar{N}}}} d x \\
= & \int_{-\infty}^{\infty} f(x) d x \\
& +\frac{\theta \sqrt{-1}}{\sqrt{\mathrm{N}}} \int_{-\infty}^{\infty} x f(x) d x \text { (which is zero as stated) } \\
& -\frac{1}{\overline{\mathrm{N}}} \frac{\theta^{2}}{2} \int_{-\infty}^{\infty} x^{2} f(x) d x \\
& + \text { terms involving } \theta^{3} \text { \&c. } \\
= & 1-\frac{1}{\overline{\mathrm{N}}} \bar{x}^{2} \frac{\theta^{2}}{2}+\text { terms involving } \theta^{3} \& \mathrm{c} . \\
= & \epsilon^{-\frac{1}{\mathbb{N}^{2}} \bar{x}^{\frac{\theta}{2}}}+\text { terms involving } \theta^{3} \& \mathrm{c} .,
\end{aligned}
$$

and therefore

$$
\mathrm{X}^{\mathrm{N}}=e^{-\overline{x^{3}} \frac{\theta^{2}}{2}}+\& \mathrm{c} \text {. }
$$


It is then shown that

$$
\begin{aligned}
& X=1 \text { if } \theta=0 \\
& X<1 \text { if } \theta \neq 0 ;
\end{aligned}
$$

and therefore in forming $X^{\mathbb{N}}, N$ being very great, we may neglect powers of $\theta$ above $\theta^{2}$, and so

$$
\mathrm{X}^{\mathrm{N}}=\epsilon^{-x^{2} \frac{\theta^{2}}{2}}
$$

and

$$
\mathrm{F}(c)=\mathrm{C} \int_{-\infty}^{\infty} d \theta \epsilon^{-\left(\overline{x^{2}} \frac{\theta^{2}}{2}+\infty \sqrt{-1}\right),}
$$

which is proportional to

$$
e^{-\frac{c^{2}}{2 x^{2}}}
$$

and the constant $\mathrm{C}$ is found from the condition that

$$
\int_{-\infty}^{\infty} \mathrm{F}(c) d c=1
$$

(2) That proposition has been extended as follows. Let $x_{1}, x_{2} \ldots x_{n}$ be $n$ mutually dependent magnitudes, and let the chance that they shall simultaneously have the values denoted by $x_{1} \ldots x_{1}+d x_{1}$, \&c., be

$$
f\left(x_{1} \ldots x_{n}\right) d x_{1} \ldots d x_{n}
$$

where $f\left(x_{1} \ldots x_{n}\right)$ is a function which vanishes for infinite values of the variables $x_{1} \ldots x_{n}$, and which is not altered when they all change sign together. Let us call the simul. taneous occurrence of $x_{1} \ldots x_{n}$ an association. The limits for each $x$ shall be $\pm \infty$. Then we have

$$
\text { Let } \begin{aligned}
\iint \ldots f\left(x_{1} \ldots x_{n}\right) d x_{1} \ldots d x_{n} & =1, \\
\iint & \ldots f\left(x_{1} \ldots x_{n}\right) x_{1} d x_{1} \ldots d x_{n}=0 \text { \&c. } \\
\iint \ldots f\left(x_{1} \ldots x_{n}\right) x_{1}^{2} d x_{1} \ldots d x_{n} & =\overline{x_{1}^{2}}, \\
\iint \ldots f\left(x_{1} \ldots x_{n}\right) x_{1} x_{2} d x_{1} \ldots d x_{n} & =\overline{x_{1} x_{2}} \text { \&c. }
\end{aligned}
$$

Let there now be $\mathrm{N}$ such associations, $\mathrm{N}$ being very large, and let each be independent of all the others. The variables in the first association shall for distinction be called $x_{11}$, $x_{12} \ldots x_{1 n}$, those in the second association $x_{21}, x_{22} \ldots x_{2 n}$, and so on to $x_{\mathrm{N} 1}, x_{\mathrm{N} 2} \ldots x_{\mathrm{N} n}$.

Phil. Mag. S. 5. Vol. 37. No. 224. Jan. 1894. L 
Let it now be required to find the chance that

$$
\begin{aligned}
& x_{1}^{\prime}=\frac{x_{11}+x_{21}+\ldots+x_{\mathrm{N} 1}}{\sqrt{\overline{\mathrm{N}}} \text { shall }}=c_{1} \ldots c_{1}+d c_{1}, \\
& x_{2}^{\prime}=\frac{x_{12}+x_{22}+\ldots+x_{\mathrm{N} 2}}{\sqrt{\mathrm{N}} \text { shall }}=c_{2} \ldots c_{2}+d c_{2}, \\
& \& \mathrm{dc.} \quad \text { \&c. } \\
& x_{n}^{\prime}=\frac{x_{1 n}+x_{2 n}+\ldots+x_{\mathbb{N} n}}{\sqrt{\mathrm{N}}} \text { shall }=c_{n} \ldots c_{n}+d c_{n},
\end{aligned}
$$

and let this chance be $\mathrm{F}\left(c_{1} \ldots c_{n}\right) d c_{1} \ldots d c_{n}$.

In the same way as for one variable $x$ we find that $\mathrm{F}\left(c_{1} \ldots c_{n}\right)=\mathrm{K} \int_{-\infty}^{\infty} d \theta_{1} \ldots \int_{-\infty}^{\infty} d \theta_{n} \mathrm{X}^{\mathbb{N}} \epsilon^{-\left(c_{1} \theta_{1}+\ldots+c_{n} \theta_{n}\right) v=1}$, in which $K$ is constant and

$$
\mathrm{X}=\int_{-\infty}^{+\infty} \int_{-\infty}^{+} \ldots f\left(x_{1} \ldots x_{n}\right) \epsilon^{\frac{1}{\sqrt{N}}\left(x_{1} \theta_{1}+\ldots+x_{n} \theta_{n}\right) \sqrt{-1}} d x_{1} \ldots d x_{n} .
$$

That is,

$$
\begin{aligned}
\mathrm{X}= & \iint \ldots f\left(x_{1} \ldots x_{n}\right) d x_{1} \ldots d x_{n} \\
& +\frac{\theta_{1} \sqrt{-1}}{\sqrt{\bar{N}}} \iint \ldots x_{1} f\left(x_{1} \ldots x_{n}\right) d x_{1} \ldots d x_{n}+\& c . \\
& -\frac{1}{\mathrm{~N}} \frac{\theta_{1}^{2}}{2} \iint \ldots x_{1}^{2} f\left(x_{1} \ldots x_{n}\right) d x_{1} \ldots d x_{n} \\
& -\frac{1}{\mathrm{~N}} \theta_{1} \theta_{2} \iint \ldots x_{1} x_{2} f\left(x_{1} \ldots x_{n}\right) d x_{1} \ldots d x_{n} \\
& -\& c .
\end{aligned}
$$

+ terms involving powers and products of $\theta_{1} \ldots \theta_{n}$ above the second degree.

All terms of the form $\iint \ldots x f\left(x_{1} \ldots x_{n}\right) d x_{1} \ldots d x_{n}$ vanish by the conditions of $f\left(x_{1} \ldots x_{n}\right)$.

The higher powers and products will be neglected for the same reason as in the case of one variable; and therefore

$$
\begin{aligned}
& \mathrm{X}=1-\frac{1}{\mathrm{~N}} \frac{\theta_{1}^{2}}{2} \overline{x_{1}^{2}}-\frac{1}{\mathrm{~N}} \theta_{1} \theta_{2} \overline{x_{1} x_{2}}+\& \mathrm{c} . \\
& =e^{-\frac{1}{\mathrm{~N}}\left(\overline{x_{1}^{2}} \frac{\theta_{1}^{2}}{2}+\overline{x_{1} x_{2}} \theta_{1} \theta_{2}+\ldots\right)},
\end{aligned}
$$

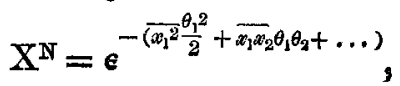


the index containing all squares and products of $\theta_{1} \ldots \theta_{n}$; and therefore $\mathrm{F}\left(c_{1} \ldots c_{n}\right)$

$$
=\mathrm{K} \iint \ldots \mathrm{e}^{-\left\{\overline{x_{1}} \frac{\theta_{2} 2}{2}+\overline{x_{1} x_{2} \theta_{1}} \theta_{2}+\ldots+\left(c_{1} \theta_{1}+\ldots+c_{n} \theta_{n}\right) \sqrt{-1}\right\}} d \theta_{1} \ldots d \theta_{n}
$$

the limits being $\pm \infty$, and $K$ being a constant to be found by making $\iint \ldots \bar{F}\left(c_{1} \ldots c_{n}\right) d c_{1} \ldots d c_{n}=1$.

(3) Now for two variables $x_{1}, x_{2}$ we find that

$$
\begin{aligned}
& \int_{-\infty}^{\infty} d \theta_{1} \int_{-\infty}^{\infty} d \theta_{2} \epsilon^{-\left\{\overline{x_{1}} \frac{\theta_{1}^{2}}{2}+\overline{x_{1} x_{2}} \theta_{1} \theta_{2}+\overline{x_{2}} \frac{\theta_{2}^{2}}{2}+\left(c_{1} \theta_{1}+c_{2} \theta_{2}\right) \sqrt{-1}\right\}}
\end{aligned}
$$

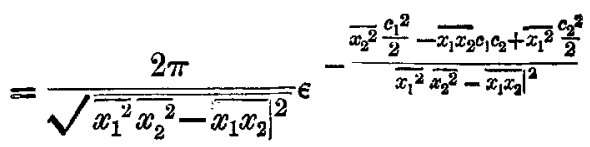

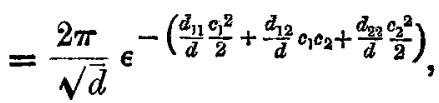

where $d$ is the determinant,

$$
d=\left|\begin{array}{ll}
\overline{x_{1}{ }^{2}}, & \overline{x_{1} x_{2}} \\
\overline{x_{1} x_{2}}, & \overline{x_{2}{ }^{2}}
\end{array}\right|
$$

and $d_{11}, d_{12}, d_{22}$ are its minors.

We infer, and it can be proved, that the same law holds for $n$ variables. That is that if

then

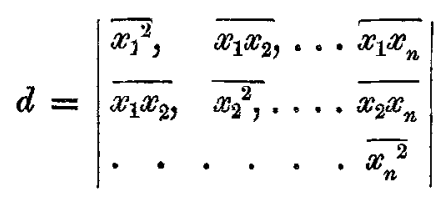

$$
\mathbf{F}\left(c_{1} \ldots c_{n}\right)=\mathrm{K} \frac{(2 \pi)^{\frac{n}{2}}}{\sqrt{\bar{d}}} \epsilon^{-\left\{\frac{d_{11} c_{1}^{2}}{d}+\frac{d_{12}}{d} \dot{c}_{1} c_{2}+\ldots\right\}},
$$

in which $d_{1 r}$ is the minor formed by omitting the first row and $r$ th column of $d$, and its sign is such that

$$
d=\overline{x_{1}^{2}} d_{11}+\sum_{r=2}^{r=n} \overline{x_{1} x_{r}} d_{1 r} \text {. }
$$

(4) Now there exists a determinant

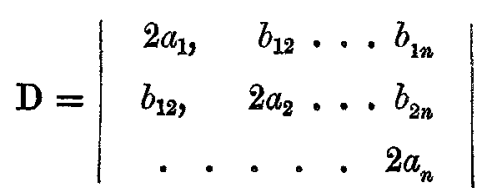

L 2 
such that

and therefore

$$
\overline{x_{1}^{2}}=\frac{\mathrm{D}_{11}}{\mathrm{D}}, \overline{x_{1} x_{2}}=\frac{\mathrm{D}_{12}}{\mathrm{D}}, \& c .
$$

$$
d=\mathbf{\Sigma} \pm \frac{\mathrm{D}_{11}}{\mathrm{D}} \quad \frac{\mathrm{D}_{22}}{\mathrm{D}} \ldots ;
$$

and thence by a known proposition * we find

$$
\frac{d_{11}}{d}=2 a_{1}, \frac{d_{12}}{d}=b_{12}, \text { \&c., and } \mathrm{D}=\frac{1}{d} ;
$$

and therefore

$$
\begin{aligned}
\mathrm{F}\left(c_{1} \ldots c_{n}\right) & =\mathrm{K}(2 \pi)^{\frac{n}{2}} \sqrt{\mathrm{D}} \cdot \epsilon^{-\left(2 a_{1} \frac{\sigma_{1}^{2}}{2}+b_{12} c_{1} c_{2}+\cdots\right)} \\
& =\mathrm{K}(2 \pi)^{\frac{n}{2}} \sqrt{\mathrm{D}} \cdot \epsilon^{-\left(a_{1} c_{1}{ }^{2}+b_{12} c_{1} c_{2}+\cdots\right)} .
\end{aligned}
$$

To determine $\mathbf{K}$ we have

$$
\iint \ldots \mathrm{F}\left(c_{1} \ldots c_{n}\right) d c_{1} \ldots d c_{n}=1 .
$$

That is, since

$$
\begin{gathered}
\iint \ldots \epsilon^{-\left(a_{1} \sigma_{1}{ }^{2}+b_{12} c_{1} c_{3}+\ldots\right)} d c_{1} \ldots d c_{n}=\frac{(2 \pi)^{\frac{n}{2}}}{\sqrt{\bar{D}}} \\
\mathrm{~K}=\frac{1}{(2 \pi)^{n}},
\end{gathered}
$$

and

$$
F\left(c_{1} \ldots c_{n}\right)=\frac{1}{(2 \pi)^{\frac{n}{2}}} \sqrt{\mathrm{D}} \cdot \epsilon^{-\left(a_{1} c_{1}^{2}+b_{12} c_{1} c_{1}+\ldots\right)} .
$$

(5) Again,

$$
\begin{aligned}
\overline{c_{1}^{2}} & =\frac{1}{(2 \pi)^{\frac{n}{2}}} \sqrt{\mathrm{D}} \iint \ldots \epsilon^{-\left(a_{1} c_{1}^{2}+b_{12} c_{1} c_{2}+\ldots\right)} c_{1}^{2} d c_{1} \ldots d c_{n} \\
& =\frac{D_{11}}{\bar{D}}=\overline{x_{1}^{2}} \text { by hypothesis. }
\end{aligned}
$$

Similarly,

and therefore

$$
\overline{c_{1} c_{2}}=\overline{x_{1} x_{2}}, \& c ;
$$

$$
\overline{\mathrm{S}}_{c}=\overline{\mathrm{S}}_{x} \text {; }
$$

and these results are true whatever be the form of the function $f\left(x_{1} \ldots x_{n}\right)$.

* Todhunter, Theory of Equations, 2nd edition, p. 393. 
If, further,

we see that

$$
f\left(x_{1} \ldots x_{n}\right)=\frac{\sqrt{\bar{D}}}{(2 \pi)^{\frac{n}{2}}} \mathrm{e}^{-\left(a_{1} x_{1}^{2}+b_{n} x_{1} x_{2}+\ldots\right)},
$$

Now let us write

$$
\mathrm{F}\left(c_{1} \ldots c_{n}\right)=f\left(c_{1} \ldots c_{n}\right) \text { 。 }
$$

$$
\begin{aligned}
& x_{1}^{\prime}=\frac{x_{11}+x_{21}+\ldots+x_{\mathrm{N} 1}}{\sqrt{\bar{N}}} \\
& x_{2}^{\prime}=\frac{x_{12}+x_{22}+\ldots+x_{\mathrm{N} 2}}{\sqrt{\bar{N}}}, \text { \&c. }
\end{aligned}
$$

Also

$$
\begin{aligned}
& \mathrm{S}_{1}=a_{1} x_{11}{ }^{2}+b_{12} x_{12} x_{12}+\ldots \\
& \mathrm{S}_{2}=a_{1} x_{21}{ }^{2}+b_{12} x_{21} x_{22}+\ldots \\
& \text { \&c. }=\& c . \\
& \mathbf{S}^{\prime}=a_{1} x_{1}^{\prime 2}+b_{12} x_{1}^{\prime} x_{2}^{\prime} .
\end{aligned}
$$

Therefore expanding $x_{1}{ }^{2}$ \&c.,

$$
S^{\prime}=\frac{S_{1}+S_{2}+\ldots+S_{N}}{N}+\text { terms containing products of }
$$

the form $x_{1 p} x_{2 q}$, that is products of two $x$ 's taken from different associations, which terms are not generally zero.

(6) The truth of the foregoing propositions will not be affected if for $a_{1}, b_{12}$, \&c. we write $\kappa a_{1}, \kappa b_{12}$, \&c., where $\kappa$ is any positive constant. In this case $\mathrm{D}$ becomes $\kappa^{n} \mathrm{D}$ and $\mathrm{S}$ becomes $\kappa \mathrm{S}$. It will be convenient if at this point w $\theta$ introduce a factor corresponding to Boltzmann's $h$, namely, $\frac{n}{2 \mathrm{~T}}$, so that henceforth we will write

$$
\begin{aligned}
& \mathrm{S}=a_{1} x_{1}^{2}+b_{12} x_{1} x_{2}+\& \mathrm{c} ., \\
& \mathrm{F}\left(x_{1} \ldots x_{n}\right)=\mathrm{Ce}^{-\frac{n \mathrm{~S}}{2 \mathrm{~T}},}
\end{aligned}
$$

where

and

$$
\mathrm{C}=\frac{\sqrt{\mathrm{D}}}{(2 \pi)^{\frac{n}{2}}}\left(\frac{n}{2 \mathrm{~T}}\right)^{\frac{n}{2}}
$$

In this case also

$$
\mathrm{D}=\Sigma \pm 2 a_{1} 2 a_{2} \& \mathrm{c} .
$$

$$
\overline{x_{1}^{2}}=\frac{2 \mathrm{~T}}{n} \frac{\mathrm{D}_{11}}{\mathrm{D}}, \quad \overline{x_{1} x_{2}}=\frac{2 \mathrm{~T}}{n} \frac{\mathrm{D}_{12}}{\mathrm{D}}, \& \mathrm{c} .
$$


and

because

$$
\begin{aligned}
\overline{\mathrm{S}} & =\mathrm{C} \iint \ldots \epsilon^{-\frac{n \mathrm{~S}}{2 \mathrm{~T}}} \mathrm{~S} d x_{1} \ldots d x_{n} \\
& =\frac{2 \mathrm{~T}}{n}\left\{a_{1} \frac{\mathrm{D}_{11}}{\mathrm{D}}+b_{12} \frac{\mathrm{D}_{12}}{\mathrm{D}}+\ldots\right\}=\frac{2 \mathrm{~T}}{n} \frac{n}{2}=\mathrm{T}
\end{aligned}
$$

$$
a_{1} \mathrm{D}_{11}+b_{12} \mathrm{D}_{12}+\ldots=\frac{n}{2} \mathrm{D}
$$

(7) We have assumed that the quadratic function

$$
\mathrm{S}=a_{1} x_{1}^{2}+b_{12} x_{1} x_{2}+\& \mathrm{c} \text {. }
$$

must have its coefficients so chosen as that it shall be positive for all values of the variables $x_{1} \ldots x_{n^{*}}$. The condition that this may be the case is that the determinant

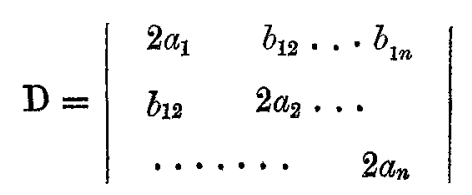

and all its coaxial minors shall be positive. Hence each of the coefficients $a$ is positive, and $4 a_{p} a_{q}-b_{p q}^{2}$ is positive for all values of $p$ and $g$.

If $\mathbf{S}$ be the energy of an association, the above condition is necessary for its stability. So for two associations near to one another, let the energy be $a_{1} x_{1}^{2}+b_{12} x_{1} x_{2}+\ldots+a_{1}^{\prime} x_{1}^{\prime 2}+\& c$, and let $\Delta$ be the double determinant $\Sigma \pm 2 a_{1}, 2 a_{2} \ldots 2 a_{1}^{\prime} \ldots$. Then in order that the two associations, considered as a single system, may be stable, $\Delta$ and all its coaxial minors must be positive. If the expression for the energy contains no terms of the form $b x x^{\prime}$, where $x$ belongs to one and $x^{\prime}$ to the other association, $\Delta$ is the product of the two single determinants $\Sigma \pm 2 a_{1}, 2 a_{2} \ldots$ and $\Sigma \pm 2 a_{1}^{\prime}, 2 a_{2}^{\prime} \ldots$, and if the condition be satisfied for them, it is satisfied for $\Delta$. But if any terms of the form $b x x^{\prime}$ make their appearance, they may make $\Delta$ or some of its coaxial minors negative, and the two associations, though separately stable; may be unstable together, and dissociation may ensue.

\section{PART II.}

(8) Now let us suppose that $\mathrm{S}$ is proportional to the kinetic energy, or to a part of the energy, of an association. Let the number of associations, or, as we will now call them, systems, be very great. Each has its own S. And we will effect a 
redistribution of $\mathbf{S}$ among them in the following way. Consider any group of $\mathrm{N}$ systems for which the variables are $x_{11}, x_{21}$, \&c., and for which $S$ has the values $S_{1}, S_{2} \ldots S_{N}$. Let these contribute respectively $\frac{S_{1}}{N}, \frac{S_{2}}{N}$, \&c.; and let the contribution $\frac{S_{1}}{N}$ be represented by the energy of a similar system whose variables are $\frac{x_{11}}{\sqrt{\mathrm{N}}}, \frac{x_{12}}{\sqrt{\bar{N}}}$, \&c. ; and let $\frac{\mathrm{S}_{2}}{\overline{\mathrm{N}}}$ \&c. be represented in the same way.

From that group of $\mathrm{N}$ sysiems we will form the variables $x_{1}^{\prime} \ldots x_{n}{ }^{\prime}$ for one system in the new distribution by making

$$
\begin{aligned}
& x_{1}^{\prime}=\frac{x_{11}+x_{21}+\ldots+x_{\mathrm{N} 1}}{\sqrt{\mathrm{N}}}, \\
& x_{2}^{\prime}=\frac{x_{12}+x_{22}+\ldots+x_{\mathrm{N} 2}}{\sqrt{\bar{N}}}, \\
& \& c . \quad \& c .
\end{aligned}
$$

see post. (11).

Now let us take at random other groups, each of $\mathrm{N}$ systems, and deal with them in the same way. And so on until every original system has appeared $\mathrm{N}$ times as a member of a group of $N$. Since in case of each group it loses $\frac{S}{N}$, it will finally have parted with all its energy $\mathrm{S}$. When this process has been carried out, the whole of $S$ for all the systems will have been redistributed among them. And owing to the mode of formation of $x_{1}^{\prime}$ \&c., the chance of the variables $x_{1}{ }^{\prime} \ldots x_{n}{ }^{\prime}$ of any system in the new distribution having the values $c_{1} \ldots c_{1}+d c_{1} \& c$. is $\mathrm{C} \epsilon^{-\frac{n \mathrm{~S}}{2 \mathrm{~T}}} d c_{1} \ldots d c_{n}$, and the aggregate energy will not have been altered.

(9) If, then, for the original systems

$$
f\left(x_{1} \ldots x_{n}\right)=\mathrm{C} \varepsilon^{-\frac{n \mathrm{~S}}{2 T}},
$$

the law of distribution is the same for the new as for the original system. In other words, it is unaffected by our process of redistribution.

If for the original systems $f\left(x_{1} \ldots x_{n}\right)$ have any other form, we shall choose $a_{1}, b_{12}$, \&c., to satisfy the equations

$$
\overline{x_{1}^{2}}=\frac{D_{11}}{D}, \quad \overline{x_{1} x_{2}}=\frac{D_{12}}{D}, \& c .,
$$


where $\mathrm{D}$ is the determinant of $\frac{n}{2 \mathrm{~T}} 2 a_{1}, \frac{n}{2 \mathrm{~T}} b_{12}$, \&c., and then in the redistributed system the law will be

$$
\mathrm{F}\left(c_{1} \ldots c_{n}\right)=\mathrm{C} \epsilon^{-\frac{n \mathrm{~S}}{2 \mathrm{~T}}}
$$

as before, and will remain unaffected by any further redistribution. In stationary motion, therefore, $\mathrm{F}\left(c_{1} \ldots c_{n}\right)$ has the form $\mathrm{C} \epsilon^{-\frac{n \mathrm{~S}}{2 \mathrm{~T}}}$, with a quadratic function for $\mathrm{S}$, whether the initial distribution $f\left(x_{1} \ldots x_{n}\right)$ has that form or not.

This assumes that the process of redistribution of the original systems has been carried out completely. If it be carried out only partially, as for instance if only a portion of the original systems be subjected to the process, the distribution in the new system will approximate more and more to the permanent form $\mathrm{C} \epsilon^{-\frac{n \mathrm{~S}}{2 \mathrm{~T}}}$, according as the redistribution is more complete. And by successive partial redistributions it will ultimately be reduced to the permanent or stationary form.

(10) The processes which we have supposed to take place successively may, of course, take place simultaneously. Further, we may suppose the whole process of redistribution to take place at a given rate per unit of time. Finally, our results will not be affected if the variables in any association undergo any other series of changes during the same time, provided these changes do not on average alter $\mathbf{S}$.

(11) By the method of art. 8 energy is conserved in the final result, but is not conserved in each separate process, because generally $\mathrm{S}^{\prime}$, the energy which we suppose to be received by a system in the new distribution, is not equal to $\frac{S_{1}+S_{2}+\ldots+S_{N}}{N}$, the energy which the contributing systems part with. That may create a difficulty in the application of the method to a physical system, even although, when the contributing systems are rapidly oscillating and in different phases, it may be that $S^{\prime}=\frac{S_{1}+S_{2}+\ldots+S_{N}}{N}$ on the average of a very short time.

(12) If our associations be material systems fixed in space, we may suppose the energy, $S$, of oscillation to be transmitted through space-i.e. æther-in waves with conservation of energy, so that the energy of a wave which passes per unit area and time through a spherical surface of radius $R$ 
described about the centre of disturbance varies as $\frac{1}{4 \pi \overline{\mathrm{R}}^{2}}$, or varies as $\frac{1}{\mathrm{~N}}$, if $\mathrm{N}=4 \pi \mathrm{R}^{2}$ and the coordinates $x$, of which that energy is a quadratic function, vary as $\frac{1}{\sqrt{N}}$. If, now, we may further assume that any number of such waves may coexist, so that the disturbance $x$ at any point at any instant is the algebraic sum of the disturbances due to all the waves which are passing the point at that instant, the redistribution of $S$ will go on in the physical system in exactly the same way as in our analytical system-and with the same result, that the distribution denoted by $\mathrm{Ce}^{-\frac{n \mathrm{~S}}{2 \mathrm{~T}}}$ will be permanent. The disturbances $x_{1} \ldots x_{n}$ in any system will be respectively $\frac{1}{2 R \sqrt{\pi}}$ times the sums of the values of the corresponding disturbances in systems distant $R$ from the one in question, as they were $t$ seconds ago, $\frac{\mathrm{R}}{t}$ being the velocity of wavemotion. The potential energy, $\chi$, of the internal forces of a system we suppose not to be capable of transmission in that form. But if the law of distribution be $\epsilon^{-h(x+s)}$, we know that this distribution will not be affected by the internal forces; neither will it be affected, on the above hypothesis, by the transmission of $\mathrm{S}$. It will therefore be permanent.

But if we are not allowed to assume the coexistence of waves in the sense above stated, the law cannot be permanent, except in the known case of binary encounters.

\section{Part III.}

(13) Onr investigation hitherto has been based on the assumption that the $\mathrm{N}$ associations are independent of one another ; that is, that the chance of the variables $x_{1} \ldots x_{n}$ in one association having assigned values is independent of the values of $x_{1}^{\prime} \ldots x_{n}^{\prime}$ the variables in another association, or, if $f(x)$ be the chance of any one of the first set having assigned value, $\frac{d f(x)}{d x^{\prime}}=0$ for each $x^{\prime}$. The condition of complete independence is that the expression for the energy contains no term of the form $b x x^{\prime}$, where $x$ and $x^{\prime}$ belong to different associations. This condition may not be satisfied if our associations are material systems very near to one another.

It is necessary to replace the stringent condition by one which can be satisfied more easily, as follows :-In the first place it will be shown that our method is applicable even 
when the associations are not all independent of each other, provided we can choose in many ways a group of them containing a great number which are independent of each other.

Let there be $\mathrm{N} q$ systems, where $\mathrm{N}$ is very large and $q$ a positive integer to which we may assign any value. Let them be called $A_{1}, A_{2} \ldots A_{N q}$. If it be not true that every one of the $\mathrm{N} q$ syste;ns is independent of every other, nevertheless it may be true, and we will assume that it is true, that the $\mathrm{N}$ systems $\mathrm{A}_{1}, \mathrm{~A}_{1+q}, \mathrm{~A}_{1+2 q}$, \&c. are independent of each other. And, similarly, the $\mathrm{N}$ systems $\mathrm{A}_{2}, \mathrm{~A}_{2+q}$, \&c. may be independent of each other, and so on for other groups. On this assumption that $A_{1}, A_{1+q}$, \&c. are mutually independent, we may apply our method to them. And let $\mathrm{F}_{1}\left(e_{1} \ldots c_{n}\right)$ $d c_{1} \ldots d c_{n}$ be the chance that the sum of the $x_{1}$ 's in these $\mathrm{N}$ systems, each divided by $\sqrt{\mathrm{N}}$, shall be $c_{1} \ldots c_{1}+d c_{1}$, \&c. Then $\mathrm{F}\left(c_{1} \ldots c_{n}\right)=\mathrm{Ce}^{-\frac{n \mathrm{~S}}{2 \mathrm{~T}}}$. Also let $\mathrm{F}_{2}\left(c_{1} \ldots c_{n}\right)$ be the corresponding chance for the group $A_{2}, A_{2+q}, \& c$. All the systems being supposed similar in character, evidently

$$
\mathrm{F}_{1}\left(c_{1} \ldots c_{n}\right)=\mathrm{F}_{2}\left(c_{1} \ldots c_{n}\right)=\text { \&c. }
$$

But the result for any one of the groups of $\mathrm{N}$ systems cannot differ from that for the whole $\mathrm{N} q$ systems. Whence it follows that $\mathrm{F}\left(c_{1} \ldots c_{n}\right)$ for the $\mathrm{N} q$ systems

$$
\begin{gathered}
=\mathrm{F}_{1}\left(c_{1} \ldots c_{n}\right)=\mathrm{F}_{2}\left(c_{1} \ldots c_{n}\right)=\& \mathrm{c} . \\
=\mathrm{C} e^{-\frac{n \mathrm{~s}}{2 \mathrm{~T}}} .
\end{gathered}
$$

We are therefore at liberty to apply our method to the $\mathrm{N}_{q}$ systems, provided that, although they are not all independent of each other, yet we can divide them into groups, each of $\mathrm{N}$ systems, the members of which are independent of one another.

(14) Again, in order that the $\mathrm{N}$ systems $A_{1}, A_{1+q}, \& c$. may be independent of each other, it will be sufficient if we make every variable, as $x$, in $\mathrm{A}_{1}$ independent of every variable $x^{\prime}$ in $\mathrm{A}_{1+q}$, \&c., that is $\frac{d f(x)}{d x^{\prime}}$ must be zero or negligible.

Let us therefore consider a series of single magnitudes $x_{1}, x_{2}$, \&c. which may be the variables in many systems of $n$, and let the chance of their simultaneously having assigned values be

$$
\mathrm{C} \epsilon^{-\frac{n}{2 \mathrm{~T}}\left(a_{1} x_{1}^{2}+b_{12} x_{3} x_{3}+8 \varepsilon \mathrm{c}\right) \text {, }}
$$

and let it be required to find the condition that $\frac{d f\left(x_{1}\right)}{d x_{r}}$ shall 
vanish. In order to find $\frac{d f\left(x_{1}\right)}{d x_{r}}$ we integrate according to each of the other variables between the limits $\pm \infty$. The result will be proportional to $\epsilon^{-\left(\mathrm{A} x_{1}{ }^{2}+\mathbf{B} x_{1} x_{n}\right)}$, where $\bar{A}$ does not concern us, and $\frac{d f\left(x_{1}\right)}{d x_{r}} \propto \mathrm{B}$, or the condition that $\frac{d f\left(x_{1}\right)}{d x_{r}}$ shall vanish is that $\mathrm{B}=0$. Also $\mathrm{B}=\frac{\Delta_{1 r}}{\Delta_{1 r r 1}}$, where $\Delta$ is the determinant of $r$ rows $\Sigma \pm 2 a_{1} 2 a_{2} \ldots$ and $\Delta_{1 r r 1}$ a coaxial minor.

(15) We have, then, to find a relation between the coefficients $a_{1}, b_{12}$, \&c., which will make $\Delta_{1 r}$ vanish compared with $\Delta_{1 r, r 1}$, without making all the coefficients $b$ vanish which connect the variables in any one of the original associations with those in any other. That can be done in many ways. It will be sufficient to give one example. Let us suppose all the $a$ 's equal to each other, and all the $b^{\prime}$ 's of the form $b_{p \overline{p+1}}$ equal to one another, and all other $b$ 's zero.

Further, let $b_{12}=b_{23}=\& \mathrm{c}$. $=2 a \theta$.

Then the determinant assumes the form

$$
\Delta=\left|\begin{array}{cccc}
2 a & b & \dot{b} & \cdot \\
b & 2 a & b & \dot{b} \\
\cdot & b & 2 a & b \\
\cdot & \cdot & b & 2 a
\end{array}\right|
$$

and if $f_{r}$ be its value for $r$ rows, the law of formation is

$$
f_{r}=f_{r-1}-\theta^{2} f_{r-2} \cdot \text {. . . . . }
$$

Since every $f$ is to be positive, $f_{r-1}>f_{r}$. Ultimately as $r$ increases the ratio $\frac{f_{r}}{f_{r-1}}$ becomes constant. Let its constant value be $\lambda$. Then equation $A$ gives

or

$$
\begin{aligned}
& \lambda=1-\frac{\theta^{2}}{\lambda}, \\
& \lambda=\frac{1}{2} \pm \frac{\sqrt{1-4 \theta^{2}}}{2},
\end{aligned}
$$

of which the positive sign will be taken. This result shows that if $f$, or $\Delta$, be always positive, $\theta^{2}$ cannot be greater than $\frac{1}{4}$, or $\theta$ greater than $\frac{1}{2}$. Also if $\theta=\frac{1}{2}$, we easily find $\Delta=\frac{r+1}{2^{r}}(2 a)^{r}$, and therefore

$$
\Delta_{1 r+1}=\frac{r-1}{2^{r-2}}(2 a)^{r-2} \text {. }
$$


Again, in this case

Therefore

$$
\begin{aligned}
\Delta_{1 r} & =\left|\begin{array}{cccc}
b & 2 a & b & \\
\cdot & b & 2 a & b \\
\cdot & \cdot & b & 2 a \\
\cdot & \cdot & \cdot & b
\end{array}\right| \\
& =(2 a)^{r-1} \theta^{r-1}=\frac{1}{2^{r-1}}(2 a)^{r-1}
\end{aligned}
$$

$$
\frac{\Delta_{1 r}}{\Delta_{1 r r 1}}=\frac{a}{r-1}
$$

and vanishes as $r$ increases indefinitely.

\section{Part IV.}

Boltzmann's Minimum Function.

(16). We have seen that, the law of distribution being $\mathrm{C} \epsilon^{-\frac{n}{2 T}}$

$$
\overline{x_{1}^{2}}=\frac{2 \mathrm{~T}}{n} \frac{\mathrm{D}_{11}}{\mathrm{D}}, \overline{x_{1} x_{2}}=\frac{2 \mathrm{~T}}{n} \frac{\mathrm{D}_{12}}{\mathrm{D}}, \text { \&c., and } \overline{\mathrm{S}}=\mathrm{T} .
$$

Let us consider a varied system in which with these same values of $\overline{x_{1}^{2}} \overline{x_{1} x_{2}}$, \&c. the law of distribution is $\mathrm{C}^{-\frac{n \mathrm{8}}{2 \mathrm{~T}}} \overline{1+q}$, where $1+q$ is any positive function of $x_{1}, x_{2}, \&$ c., and $q$ must satisfy the following conditions, namely :-

(1) In order that the number of systems may be the same in the varied as in the normal state,

$$
\begin{gathered}
\iint \ldots e^{-\frac{n \mathrm{~S}}{2 \mathrm{~T}}} d x_{1} \ldots d x_{n}=\iint \ldots \varepsilon^{-\frac{n \mathrm{~S}}{2 \mathrm{~T}}} \overline{1+q} d x_{1} \ldots d x_{n} \\
\iint \ldots q \epsilon^{-\frac{n \mathrm{~S}}{2 \mathrm{~T}}} d x_{1} \ldots d x_{n}=0 .
\end{gathered}
$$

(2) In order that the values of $\overline{x_{1}^{2}}, \overline{x_{1} x_{2}}$, \&c., may not be altered,

$$
\iint \ldots \epsilon^{-\frac{n s}{2 T}} x_{1}^{2} d x_{1} \ldots d x_{n}=\iint \ldots \epsilon^{-\frac{n \mathrm{~S}}{2 \mathrm{~T}} \overline{1+q} x_{1}^{2}} d x_{1} \ldots d x
$$

and therefore also

$$
\iint \ldots e^{-\frac{n \mathrm{~S}}{2 T} \mathrm{~S}} d x_{1} \ldots d x_{n}=\iint \ldots \epsilon^{-\frac{n \mathrm{~S}}{2 \mathrm{~T}}} \overline{1+q} \mathrm{~S} d x_{1} \ldots d x_{n} .
$$


These conditions being satisfied the system will, on free interchange of energy, pass out of the varied state into the normal state. And it can be now shown that the function $B$

$$
=\iint \ldots f\left(x_{1} \ldots x_{n}\right)\left\{\log f\left(x_{1} \ldots x_{n}\right)-1\right\} d x_{1} \ldots d x_{n}
$$

diminishes in the process.

For let $B_{0}$ be the value of $B$ in the normal state, when $f\left(x_{1} \ldots x_{n}\right)=\mathrm{Ce}^{-\frac{n \mathrm{~S}}{2 \mathrm{~T}}}, \mathrm{~B}$ its value when

\section{Then $\mathrm{B}-\mathrm{B}_{0}=$}

$$
f\left(x_{1} \ldots x_{n}\right)=\mathrm{C}^{-\frac{n \mathrm{~s}}{2 \mathrm{~T}}} \overline{1+q} .
$$

$$
\begin{aligned}
\mathrm{C} \iint & \ldots \mathrm{e}^{-\frac{n \mathrm{~S}}{2 \mathrm{~T}}} \overline{1+q}\left(\log \mathrm{C}-\frac{n \mathrm{~S}}{2 \mathrm{~T}}-1+\log \overline{1+q}\right) d x_{1} \ldots d x_{n} \\
& -\mathrm{C} \iint \ldots \epsilon^{-\frac{n \mathrm{~S}}{21^{1}}}\left(\log \mathrm{C}-\frac{n \mathrm{~S}}{2 \mathrm{~T}}-1\right) d x_{1} \ldots d x_{x_{n}} \\
& =\mathrm{C} \iint \ldots \epsilon^{-\frac{n \mathrm{~S}}{2 \mathrm{~T}}} \overline{1+q} \log \overline{1+q} d x_{1} \ldots d x_{n} \\
& =\mathrm{C} \iint \ldots \epsilon^{-\frac{n \mathrm{~S}}{2 \mathrm{~T}}}\{\overline{1+q} \log \overline{1+q}-q\} d x_{1} \ldots d x_{n},
\end{aligned}
$$

because

$$
\mathrm{C} \iint \ldots \mathrm{e}^{-\frac{n \mathrm{~s}}{2 \mathrm{~T}}} q d x_{1} \ldots d x_{n}=0 .
$$

Now since $1+q$ is positive, $\overline{1+q} \log \overline{1+q}-q$ is necessarily positive, unless $q=0$, -and is then zero; $\mathrm{B}-\mathrm{B}_{0}$ is therefore positive. And given $\mathrm{T}$ and the coefficients $a_{1}, b_{12}$, \&c., $\mathrm{B}$ has its least possible value when $q=0$, or $f\left(x_{1} \ldots x_{n}\right)=\mathrm{Ce}^{-}-\frac{n \mathbf{S}}{2 \mathrm{~T}}$.

And this least possible or minimum value differs by a constant from $\log C$ or $\log \left\{\left(\frac{n}{2 \mathrm{~T}}\right)^{\frac{n}{2}} \frac{\sqrt{\mathrm{D}}}{(2 \pi)^{\frac{n}{2}}}\right\}$.

Further, $\frac{d}{d q}\left(\mathrm{~B}-\mathrm{B}_{0}\right)=\log (1+q)$, and therefore $\mathrm{B}-\mathrm{B}_{0}$ diminishes as $q$ approaches zero.

The Second Law of Thermodynamics.

(17) In stationary motion the minimum function has the value

$$
\mathrm{B}=\left(\frac{n}{2 \mathrm{~T}}\right)^{\frac{n}{2}} \frac{\sqrt{\mathrm{D}}}{(2 \pi)^{\frac{n}{2}}} \text { plus a constant. }
$$

It is a function of $\mathrm{T}$ and the parameters $a_{1}, b_{12}$, \&c., or any 
parameters $v_{1}, v_{2}$, \&c., on which $a_{1}, b_{12}$, \&c., depend. The second law may from one point of view be regarded as the law of the variation of $B$ when $T$ and the parameters vary very slowly, so that stationary motion is always attained. On this assumption the proofs of the second law depend.

We have seen that $\bar{S}=\mathrm{T}$ and is independent of the parameters, or $\frac{d}{d v} \overline{\mathrm{S}}=0$ for each $v$. But $\frac{\overline{d S}}{d v}$ is not generally zero. It may, therefore, be the case that work has to be done on variation of any parameter $v$. This work will be denoted by $-\frac{\overline{d S}}{d v} \partial v$. It will include the work done against all external forces. The energy imparted during any small variation of $T$ and the parameters will be denoted by $\partial Q$. Then the second law requires that $\frac{\partial Q}{\mathrm{~T}}$ shall be a complete differential.

(18) It will be sufficient to prove the law for any parameter $v$ on which $a_{1}, b_{12}$, \&c. depend. So far as this proof is concerned, there may be many such.

We have in this case

$$
\begin{aligned}
\partial \mathrm{Q} & =\partial \mathrm{T}-\frac{\overline{d \overline{\mathrm{S}}}}{d v} \partial v \\
& =\partial \mathrm{T}-\mathrm{C} \partial \iint \epsilon^{-\frac{n \mathrm{~S}}{2 \mathrm{~T}}} \frac{d \mathrm{~S}}{d v} d x_{1} \ldots d x_{n} \\
& =\partial \mathrm{T}-\mathrm{C} \partial \iint \ldots \epsilon^{-\frac{n \mathrm{~S}}{2 \mathrm{~T}}}\left\{\frac{d a_{1}}{d v} x_{1}^{2}+\frac{d b_{12}}{d v} x_{1} x_{2}+\ldots\right\} d x_{1} \ldots d x_{n} \\
& =\partial \mathrm{T}-\partial v \frac{2 \mathrm{~T}}{n}\left\{\frac{d a_{1}}{d v} \frac{\mathrm{D}_{11}}{\mathrm{D}}+\frac{d b_{12}}{d v} \frac{\mathrm{D}_{12}}{\mathrm{D}}+\ldots\right\} \\
& =\partial \mathrm{T}-\partial v \frac{2 \mathrm{~T}}{n} \frac{1}{2}\left\{\frac{d a_{1}}{1} \frac{1}{d v} \overline{\mathrm{D}} \frac{\mathrm{D}}{d a_{1}}+\frac{d b_{12}}{d v} \frac{1}{\mathrm{D}} \frac{d \mathrm{D}}{d b_{12}}+\ldots\right\} \\
& =\partial \mathrm{T}-\partial v \frac{\mathrm{T}}{n} \frac{1}{\mathrm{D}} \frac{d \mathrm{D}}{d v} . \\
\frac{\partial \mathrm{Q}}{\mathrm{T}} & =\partial \log \mathrm{T}-\frac{1}{n} \partial \log \mathrm{D} .
\end{aligned}
$$

Now in this case,

$$
\begin{aligned}
\partial \mathrm{B} & =\partial \log \left\{\sqrt{\mathrm{D}}\left(\frac{n}{2 \mathrm{~T}}\right)^{\frac{n}{2}}\right\} ; \\
\therefore \quad \frac{1}{n} \partial \log \mathrm{D} & =\frac{2}{n} \partial \mathrm{B}+\partial \log \mathrm{T} ; \\
\therefore \quad \frac{\partial \mathrm{Q}}{\mathrm{T}} & =-\frac{2}{n} \partial \mathrm{B} .
\end{aligned}
$$

